\title{
The influence of external climatic factors on the accuracy of the forecast of energy consumption
}

\author{
J.E. Shklyarskiy and D.E. Batueva* \\ Saint Petersburg Mining University, St. Petersburg, Russian Federation
}

\begin{abstract}
The object of the study is the territory, which is characterized by household and production load, and their characteristic tendencies of changes in the volume of electricity consumption, and therefore it is necessary to create forecast models that take into account the influence of external climatic fac-tors and their contribution to the forecast of energy consumption of the ob-ject. In the work, theoretical methods and experimental studies were used, consisting in a scientific analysis of trends in changes in power consumption depending on changes in factors, methods of mathematical statistics, statis-tical samples, factors and data from the weather service. During this study, external climatic factors that influence the process of changing the energy consumption of an object and their degree of influence on changing consumption were determined. To improve the accuracy of forecasting, it is pro-posed to break the data into working days and days off, since consumption in these periods is of a different nature.
\end{abstract}

\section{Introduction}

The main factor for a power supply company when calculating the final price of electricity for consumers is the cost of its purchase in the market "one day ahead". At the same time, the commercial interest of the wholesale electricity market entities is the use and development of relevant methods and infrastructure automated control and accounting systems for electricity, as well as the use of modern software with a single database that reflects all the company's business processes. Prices on the balancing market, as a rule, differ from prices on the day-ahead market by $10-30 \%$ in the direction of increase in the Russian Federation, this indicator differs by 2-5 times in the leading countries for equipping with automated commercial electricity metering systems (Canada, France, Germany), where there are no problems with the collection of interval readings of metering devices, which carries increased requirements for the construction of predictive models of electricity consumption.

The current model of the wholesale electricity market in the Russian Federation defines the rules for the purchase of the required amount of electricity for subjects as follows: experts make a forecast in the "day ahead" mode using established techniques and information systems, then an application is sent to the "Trading System Administrator" for the purchase of the required volumes of electricity for day-ahead market for every hour of the next day. The cost of a kilowatt-hour of search or shortage of the submitted value is calculated according to the prevailing fact - according to the tariff of the balancing market. In this regard, the importance of the process of short-term / medium-term forecasting of the required volumes of electric energy in the "dayahead market” and the formation of a medium-term plan for the power supply company is growing.

It is necessary to create a model that allows predicting the energy consumption of facilities by analogy with the wholesale electricity market, located, for example, in the Arctic, powered by diesel generator sets and wind turbines, especially in connection with the sharply changing nature of climatic and meteorological conditions. The deviation of real energy consumption from the curve of the day's workload is from $11 \%$ to $56 \%$ in the Arctic, with the maximum deviation occurring in the summer months. This is because the winter is consistently low air temperatures and the average temperature changes significantly every month in the spring-summer and autumn periods, which entails a shift in the load curve. As a result, the deviation of real loads from the energy balances used in calculations for the summer regime day can be $40-50 \%$.

In view of the above, each object needs to have a database of retrospective data and proven methodological models for constructing shortterm/medium-term forecasts of power consumption, with the goal of planning the largest amount of electricity needed, the wind turbine and diesel generator sets working together and ensuring the smallest deviations from the predicted values. Unfortunately, to date, a unified and generally accepted methodological base has not been created. In this regard, the development of a methodological base for creating prognostic models is an urgent task.

* Corresponding author: dasha-batueva4@rambler.ru 
Currently, many researchers use neural networks in their work [1-4]. They note good predictive abilities and the ability to dynamically adapt to changing conditions, but the disadvantage is the difficulty in determining the optimal configuration and the optimal set of input parameters of the neural network [5-6]. Each object requires a different set of data and external factors that affect energy consumption to a different extent [7]. In addition, most of the above publications justify the possibility of predicting power consumption using neural networks, without presenting any practical recommendations or techniques for the formation of existing forecast models based on them.

The aim of the work is to determine the external climatic factors and their contribution in predicting the energy consumption of an object. Accurate prediction of energy consumption is an effective tool for energy saving and increasing the energy efficiency of electric energy consumption [8]. However, the accuracy of the forecast depends on the amount of input data.

Based on the foregoing, the objectives of the study are the analysis of factors affecting energy consumption, to determine those that directly affect the change in energy consumption, and it is also necessary to explore the possibilities of different data combinations taking into account the time of day, day of the week and season of the year to obtain the minimum inaccuracies.

\section{Methods}

Currently, many different approaches, methods and models for predicting energy consumption for the short, medium and long term are used, but each approach has its own characteristics [9-13].

Today, the most popular statistical forecasting methods, which provide for the possibility of using a large number of different input parameters, and the function of the influence of the input parameter on the output result can be of any complexity - non-linear, nonstationary, etc [14]. In addition, not all parameters are measured in the same units: some input parameters of the model are numerical (historical values of electricity consumption, time of day, air temperature, etc.), and some parameters are categorical (cloud type, season, day type, and other) [15]. Implicit inclusion of input parameters may be used. For example, the construction of an additional system, each element of which calculates for a specific case, for example, for a specific type of day or time of year.

Theoretical methods and experimental studies were used in the study, which consisted in a scientific analysis of the trends in the volume of electricity consumption depending on changes in factors, methods of mathematical statistics, statistical samples, factors and data from the weather service.

\subsection{Electricity consumption trends for various types of consumers}

It should be borne in mind that for each group of consumers, such as the population or industrial facilities, their own trends in changes in the volume of electricity consumption are characteristic. Industrial enterprises, which are characterized by a five-day work week, consume less on holidays and weekends. In addition, consumption is not uniform throughout the week, after weekends and holidays there is a decline in consumption, by the middle of the week there is an increase in electricity consumption.

Power consumption also changes in the winter and summer periods, which is associated with the inclusion of additional equipment inherent in this season. Residential consumers have their own nature of consumption: heating systems are used in winter, the summer season opens in summer, and there is also an increase in electricity consumption on holidays and weekends. These patterns are basic for these consumer groups, but should be investigated more accurately at a specific facility.

Analysis of various types of activities in Russia and foreign countries shows that enterprises have increased energy intensity in Russia. This is due to many influencing factors: natural and climatic conditions, the average annual temperatures are usually low for the Russian climate, the consumption of fuel and energy resources for reliable and sustainable energy supply to consumers is growing at low temperatures, in addition, many large industrial centers are remote from each other, which leads to increased energy costs to ensure the exchange of services and goods between these regions.

\subsection{Forecasting with a changing process of energy consumption}

There are a number of factors and various dependencies in enterprises that are difficult to consider when designing [16-18]. Thus, the operating modes and schedules of industrial enterprises are very diverse; vary over time with a natural tendency for continuous growth of electrical loads, accordingly, it is necessary to develop a methodology for efficient and accurate planning of energy consumption, which can be done on the basis of neural network forecasting. It will be necessary to take into account a number of external factors in the process of building the model, which vary in their laws, which can affect the load schedules of consumers. Consumer load can vary depending on various influencing factors, such as: weather and climate, time of day, month of the year, geographical location and economic factors.

The review revealed that the number of factors may include (depending on the

1. Environmental parameters (air temperature, precipitation, cloud cover, wind speed and direction).

2. The volume of output, start/stop of technological chains.

3. Price situation in the electricity market (if it is possible to regulate the load schedule). 
4. Carrying out scheduled and unscheduled outages, repairs.

5. Emergencies, malfunctions in the work of technological chains.

The optimal forecast model should take into account as many influencing factors as possible. In addition, factors can be interdependent [19-20]. The influence of environmental parameters on the energy consumption process in this work is investigated. It is quite difficult to determine the "contribution" of each factor to the change in the amount of electricity consumed in a complex, constantly changing system and is one of the objectives of this study. In the study of existing statistical methods, it was found that there is no suitable method that could take into account all the features of a particular enterprise and factors that have a significant impact on the change in electricity consumption.

\subsection{Parameters for modeling power consumption}

The power consumption process is a complex random non-stationary process, which can be represented by several regular and irregular (random) components. By the method of modeling power consumption as a random non-stationary process, the initial process is divided into regular (trend) and irregular components. A trend describes steady trends in a process over time. The irregular component characterizes the random unpredictable part of consumption and the probable deviations of the actual values of energy consumption from the trend identified from the initial process.

The error is determined between the initial data and the results of the mathematical model to assess the adequacy of the model. In the general case, the predictive mathematical model of power consumption may include elementary functions of any independent variables taken as factors affecting the amount of consumption, for which there is reliable evidence, as well as forecasts obtained from official sources. The model allows the use of the following independent variables or influencing factors; seasonality, daylight hours, daily ambient temperature graphs.

\subsection{Construction of the correlation dependence of factors}

Long-term and short-term weather forecasts, including meteorological parameters necessary for use in the process of forecasting power consumption, are taken from the archive data of the Hydrometeorological Center of Russia.

The source data was taken hourly consumption by the object for three years for research. It was revealed after analysis of the initial data that the data have big differences on working days, weekends and holidays. The general trend of energy consumption was clearly visible: on Monday - underestimated consumption, by Wednesday - an increase in consumption, by Friday - a decline, and on weekends and holidays, consumption is low. Seasonality was also clearly visible: in the winter months, electricity consumption grew, in the summer months there was a decline. This is due to the fact that during the winter months, electricity consumption increases for the heating process. In this regard, a separate forecasting model was built for each day of the week, holidays and weekends are combined into one model.

When choosing the factors that will be used in predicting the volume of electricity, those were expertly identified that could presumably influence the process under study. As a result of expert selection of factors, it was decided to leave the following:

- day of the week;

- seasonality;

- daylight hours;

- meteorological factors for each day (ambient temperature, cloudiness, humidity, pressure);

- electricity consumption (kWh.)

Next, a correlation matrix was built to identify factors that have a significant impact on electricity consumption. Using a paired linear correlation coefficient, the tightness of the relationship between the two features is measured. The linear correlation coefficient is calculated by the formula 1 :

$$
r_{x y}=\frac{\sum_{i=1}^{n}\left(x_{i}-\bar{x}\right)\left(y_{i}-\bar{y}\right)}{\sqrt{\sum_{i=1}^{n}\left(x_{i}-\bar{x}\right)^{2}\left(y_{i}-\bar{y}\right)^{2}}}
$$

where $x_{i}$ and $y_{i}$ are the values of the factors $\mathrm{x}$ and $\mathrm{y}$, respectively, for the $\mathrm{i}$-th object, $i=1, . ., n$; $n$ is the number of objects; $\bar{x}$ and $\bar{y}$ - arithmetic mean values of signs $x$ and $y$, respectively.

The linear correlation coefficient varies from -1 to +1 . Equality of the coefficient to zero indicates the absence of a linear relationship. The equality of the coefficient -1 or +1 indicates the presence of a functional relationship (i.e., a change in one phenomenon is accompanied by a change in another).

The STATISTICA software product allows you to display the correlation dependence in the form of a graph to clearly identify the factors that influence the indicator "consumption of electricity volumes". An analysis of the correlation of selected factors is presented in Figure 1.

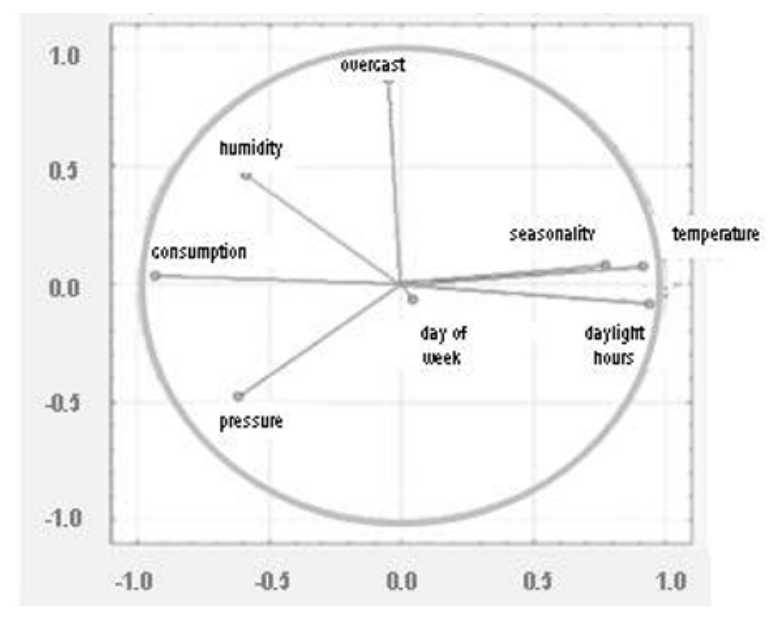

Fig. 1. Correlation of factors. 
Factors that directly affect the change in data volumes lie on the other side of the axis from the desired indicator. An analysis of the correlation of factors shows that seasonality, ambient air temperature and daylight hours directly affect the change in electricity volumes. Based on this data, electricity consumption can be predicted.

\section{Results and Discussion}

It was revealed as a result of data analysis that the most effective model is obtained by dividing the initial data on environmental parameters by days of the week. This is due to the fact that a large share of energy consumption falls on the enterprises of the studied territory, including very large ones: factories, various industries. The main peak of electricity consumption for enterprises falls in the middle of the week, machines and workshops consume very little electricity on weekends, production machines and furnaces are heated at the beginning of the week and electricity consumption increases. The factor of seasonality is of the greatest importance for electricity consumption among the population; in summer, electricity consumption in gardening associations, recreation centers and so on.

\section{Conclusions}

1. It was revealed as a result of the study that forecasting power consumption is an effective tool for energy conservation and improving the energy efficiency of electricity consumption.

2. An analysis of factors affecting energy consumption showed that the ambient temperature directly affects the change in energy consumption. A significant influence is exerted by factors: a sign of seasonality, daylight hours. Insignificant influence is exerted by factors of pressure, cloudiness, rainfall. The humidity factor does not affect the change in power consumption.

3. It is proposed to break the data into working days and weekends to increase the accuracy of forecasting. Weekends and holidays can be combined into one group.

\section{References}

1.X. Zhang, J. Yuan: Electricity Consumption Forecasting Based on Improved BP Neural Network. In: 2008 International Conference on Risk Management \& Engineering Management, 357-360. IEEE, China (2008)

2.Y.Y. Xu, R. Hsieh, Y.L. Lu, Y.C. Shen, S.C. Chuang, H.C. Fu, C. Bock, H.T. Pao, Forecasting electricity market prices: a neural network based approach. In: 2004 IEEE International Joint Conference on Neural Networks, 4, 2789-2794. IEEE, Hungary (2004)

3.H. Wezenberg and M. B. Dewe, Adaptive neural networks for tariff forecasting and energy management. In: Proceedings of ICNN'95 - International Conference on Neural Networks, 2, 877-881 (1995)
4. M. Gavrilas, S. Member, O. Ivanov, G. Gavrilas, Customer Classification and Load Profiling using Data from Smart Meters. In: 12th Symp. Neural Netw. Appl. Electr. Eng., 1-6. Serbia (2014)

5. A. Bala, N. K. Yadav, N. Hooda, D. Registrar, Implementation of Artificial Neural Network for Short Term Load Forecasting. In: Curr. Trends Technol. sci., 3(4), 247-251 (2014)

6.D. Kown, M. Kim, C. Hong, S. Cho, Short term load forecasting based on BPL neural network with weather factors. In: Int. J. Media Ubiquitous Eng., 9(1), 415-424 (2014)

7.V.Y. Koptev A.V. Kopteva D.A. Poddubniy, Increase in Energy Efficiency of Oil and Gas Companies by Perfecting of Management Systems. In: 2019 IEEE Conference of Russian Young Researchers in Electrical and Electronic Engineering (EIConRus 2019), 548-552 IEEE, Russian Federation (2019)

8. Mat Alejandro, A hybrid integrated architecture for energy consumption prediction. In: Future Generation Computer Systems, 63, 131-147. The Netherlands (2016)

9.D. Kryukov, M. Agafonova, A. Arestova, Comparison of regression and neural network approaches to forecast daily power consumption. In: 2016 11th International Forum on Strategic Technology (IFOST), 247-250. Russian Federation (2016)

10. Q. Ahsan and M. Uddin: A Probabilistic Approach of Electrical Energy Forecasting. In: 2005 IEEE Instrumentation and Measurement Technology Conference Proceedings, 1070-1074. IEEE, Canada (2005)

11. N. J. Hobbs, B. H. Kim and K. Y. Lee, Longterm load forecasting using system type neural network architecture. In: 2007 International Conference on Intelligent Systems Applications to Power Systems, 1-7 IEEE, Japan (2007)

12. Y. Ma, P. Liu, Y. Cui, S. Liu, Proposed Model Employing ARIMA and RELM in Urban Energy Consumption Prediction. In: 2018 International Symposium on Computer, Consumer and Control (IS3C), 465-468. IEEE, Taiwan (2018)

13. B. F. Ummuhan, O. N. Gerek, M. Kurban, A novel modeling approach for hourly forecasting of longterm electric energy demand. In: Energy Convers. Manag., 52, 199-211. Turkey (2011)

14. D. Kown, M. Kim, C. Hong, S. Cho, Short term load forecasting based on BPL neural network with weather factors. In: Int. J. Media Ubiquitous Eng., 9(1), 415-424 (2014)

15. M. Mustapha, M. W. Mustafa, S. N. Khalid, I. Abubakar and H. Shareef: Classification of electricity load forecasting based on the factors influencing the load consumption and methods used: An-overview. In: 2015 IEEE Conference on Energy Conversion (CENCON), 442-447 IEEE, Malaysia (2015)

16. Iu. Murashov, V. Frolov, D. Uhrlandt, S. Gorchakov, D. Ivanov, A. Sivaev, Analysis of arc processes in multi-chamber arrester for lightning protection at high-oltage overhead power lines. In: Plasma Physics and Technology Journal, 4, 124-128. Czech Republic (2017) 
17. I.S. Churkin, D. Ivanov, V. Frolov, D. Uhrlandt, Improvement of the efficiency $\mathrm{f}$ an ar plasma spraying technology. In: 19th Symposium on Physics of Switching Arc, FSO 2011, 149-152 Czech Republic (2011)

18. J. Zhu, The Optimization Selection of Correlative Factors for Long-Term Power Load Forecasting. In: IEEE 5th Internatinal Conf. HumanMachine Sys. Cybern., 1, 241-244 IEEE, China (2013)

19. I. Murashov, V. Frolov, A. Kadyrov, Development of the arc plasma torch operation mathematical model for spheroidization of finedispersed powders. In: Journal of Physics: Conference Series, 1058(1), 012024 IOP Publishing, Russian Federation (2018)

20. A. Chusov, G.V. Podporkin, M. Pinchuk, D. Ivanov, I. Murashov, V. Frolov, Development of a physical 2-D model for arc quenching chamber of lightning protection multichamber system. In: 33rd International Conference on Lightning Protection (ICLP), 1-9 Portugal (2016) 